\title{
Infant valsalva retinopathy: A re-interpretation of the 1992 Sheffield study of eyes in Shaken Baby Syndrome cases
}

Talbert DG*

Institute of Reproductive and Developmental Biology, Imperial College School of Medicine, Queen Charlotte's Hospital, UK

\begin{abstract}
Introduction: Retinopathy is a key factor in the triad of findings for convictions for Shaken Baby Syndrome. It is generally believed to result from inertia effects during manual shaking of the infant.

The Sheffield study of non-accidental head injuries: A detailed study of the eyes of children identified as having died from non-accidental cerebral causes, was carried out by the University of Sheffield (1988-1992). They concluded that haemorrhages and retinal detachments were caused by vitreous traction forces caused by movement of the vitreous during shaking. However, Duane showed that retinopathy can result from raised venous pressure during actions causing raised thoracoabdominal pressure (Valsalva Retinopathy). Blood is forced up the jugular veins and, depending of the strength of the individual's jugular vein valves, may be forced into cerebral veins. This study applies Duane's findings in adults to infants.
\end{abstract}

Analysis: (1) Individual vulnerability depends on the strength of the inner jugular vein valves, which is known to vary.

(2) Subdural and retinal hemorrhages have a common factor, venous surge pressure. They are not independent.

(3) The lower incidence of injuries at the ocular equator is related to the reduced diameter of retinal veins and Laplace's P=T/r Law

(4) The high incidence of hemorrhages and detachments at the Ora Surrata/Retina boundary is of anterior ocular venous blood from the copious ciliary blood supply, not the retinal veins.

The precise details of the intraocular hemorhages and detachments given in the Sheffield study fit well with those anticipated from sudden surges of intracranial venous pressure such as those associated with pyloric stenosis vomiting.

Conclusion: The Sheffield intraocular data fits better with an infant form of Duane's Valsalva Retinopathy than with Shaking. The Triad (Subdural Hemorrhage, Retinal Hemorrhage, and Encephalopathy) are not independent variables, they are manifestations of a common Venous Hypertension.

\section{Introduction}

There is a disorder that results in infants presenting at hospital emergency departments with intracranial and intraocular bleeding, without visible trauma to the head. Since there is no external evidence of injury, it is currently believed that these injuries result from shaking of the infant by the parent or carer. It is believed that the co-incidence of three forms of injury, Intracranial (Subdural), Retinopathy, and Central Nervous System (CNS) malfunction, known as the "Triad", constitute proof of shaking. The Sheffield study concentrated on the nature of ocular injuries in infants diagnosed as having been injured by shaking.

\section{Sheffield study}

In the following italics are used to indicate direct quotes from sources.

In 1996, Green et al. [1] made a valuable contribution to understanding the incidence of injuries, by ranking the various forms of injury. The eyes of 23 children, diagnosed as having nonaccidental injuries were examined, initially in situ and then after removal of the roof of the orbit, to determine the extent of orbital and periocular traumatic abnormalities, and to establish the extent of orbital and perineural (subdural) haemorrhage into the optic nerve sheath. Following removal the eyes were... fixed in formaldehyde, then alcohol....They were then embedded in paraffin wax, and five horizontally orientated haematoxylin and eosin sections through the centres of the optic nerve and pupil prepared. We recorded traumatic ocular lesions by determining the presence or absence in one or both eyes of retinal detachment,... and vitreous, subhyaloid, intraretinal, choroidal, and perineural (optic nerve) haemorrhages....

All but one of 16 infants whose death was attributed to head injury had subdural haemorrhage (94\%); in eight of these (53\%) subdural hemorrhage was the only localised injury....

There is a strong association between CNS injury and traumatic ocular lesions. Eye injuries were present in $81 \%$ of the 16 children who died of head injuries but only $17 \%$... of those whose death was due to other causes...

The incidences of subhyaloid, intraretinal, and perineural (optic) nerve hemorrhages were significantly increased in children who died as a

Correspondence to: Talbert D.G., Institute of Reproductive and Developmental Biology, Imperial College School of Medicine, Queen Charlotte's Hospital, Du Cane Road, London W12 ONN, UK, E-mail: d.talbert@imperial.ac.uk

Received: February 17, 2017; Accepted: February 25, 2017; Published: February 28,2017 
result of CNS trauma, and in those with subdural hemorrhage. Although all of the children with perineural (subdural) optic nerve (sheath) hemorrhages had co-existing subdural hemorrhages, the perineural hemorrhages involved only the distal segment of the nerve, and did not involve the proximal part of the optic nerve within the apex of the orbit, confirming that there was no direct connection between these two separate sites of hemorrhage.

Their results also clearly showed a consistent order of forms of injury which they attributed increasing degrees of shaking trauma.

\section{Valsalva retinopathy}

The purpose of this present study was to apply the concept of venous hypertension surge, (such as that occurring with pyloric stenosis vomiting and retching) to interpreting the Sheffield study findings of retinopathy in cases diagnosed as due to shaking trauma [1].

Duane is credited with introducing the concept of "Valsalva Hemorrhagic Retinopathy". In 1972 he presented it at a seminal meeting [2] as "any rise in intrathoracic or intra-abdominal pressures particularly against a closed glottis (coughing, vomiting, lifting, straining at stool, wind blast injuries...) are all expressions of the well known Valsalva maneuver which produces a reflux venous pressure rise in the upper reaches of the body. Vomiting in Infant Pyloric Stenosis is one such causative agent.

Duane commented that the fact that "the fundus, especially the retina, responds to alterations of homeostasis produced by distant trauma has been recognised since shortly after the discovery of the opthalmoscope."....

Whooping cough is a natural model for the effects forced expiratory efforts. Before the introduction of immunisation mechanical damage could be quite severe. McKendrick [3], writing in 1978, stated "Mechanical complications occur solely in the paroxysmal stage as a result of increased pressure in the thorax, abdomen or skull. They appear during or immediately following a severe spasm. Bleeding may occur from any surface or into the brain but the commonest results of rupture of blood vessels are epistaxis, subconjunctival hemorrhage, and petechiae in the skin over the neck and upper chest. Hemoptysis, retinal detachment, and bleeding from the ears have all occurred. Herniae or rectal prolapse may appear.

\section{Valsalva retinopathy in adults}

Duane reported a personal communication from a Dr Hoyt of cases of Valsalva retinopathy, "a boy who was doing bench presses with 175-pound weights (Case 1), a girl water skier who held her breath as she was flipped by a wave (Case2), a lady who was helping a neighbour move her refrigerator down a flight of stairs (Case 3). All these patients had a variable but pure hemorrhagic retinopathy, most of which was preretinal and on occasion even extended into the vitreous.

At this meeting a Dr Norton reported a 33-yearold male (Case 4), who awoke one morning with a headache. He had been at a party the night before. He vomited, and when he vomited, he suddenly noticed loss of vision in his right eye. Three weeks later his vision was around $20 / 100$. Over the next 3 months his right eye improved to 20/20.

Dr Norton also reported a woman of 27 in her eighth month of pregnancy (Case 5) who woke up with a coughing spell. She then found she could not see out of one eye. A few hours later her vision acuity was measured as 20/400. After 3 months her vision was back to 20/20.
Another case was a 33-year-old physician (Case 6) who, while having a violent argument with his girl friend suddenly realised the vision in one eye had gone, yet subsequently he recovered completely 20/20 vision

A Dr Kearns reported a 15 year-old boy (Case 7) who developed a hemorrhage while blowing up an air mattress, and a $22 \mathrm{yr}$ old man (Case 8) who woke up with blurred vision in his left eye attributed to "dry Heaves".

Other cases from the literature are:-

Weight lifting [4]: A healthy 17-year old (Case 9) with sudden visual loss in his left eye following weight lifting. Corrected visual acuity was 20/20 in the right eye and 20/200 in the left. Ophalmoscopy of the left fundus revealed "cotton-wool" spots and superficial retinal haemorrhages in the macular and peripapillary areas.

Back slapping [5]: A 2 month old boy (Case 10) was discovered in his pram pallid and limp. The mother held the baby to her shoulder and repeatedly slapped him on the back, and he began to breathe again. There was a little blood staining on the face and pillow, and some bloody mucus was expelled from the nostrils. Extensive fresh haemorrhages were found in the nerve fibre layer of both fundi, and bilateral macular oedema but no swelling of the optic discs. Chest radiography showed patchy perihilar shadowing. They comment "Retinal haemorrhages can be produced by thoracic compression that is insufficient to cause detectable damage to the chest itself" "We think that this baby became apnoeic after aspirating blood from an epistaxis, and that the mother's life saving measures transmitted pressure from the thorax to retinal veins that were already compromised by hypoxia."

Trombonist [6]: A $50 \mathrm{yr}$ old man (Case 11), solo trombonist for 26 years, ( play + practice 6 hrs a day) noted a central scotoma in the right eye after playing a strenuous musical piece by Wagner (Scotoma $=$ a region of diminished acuity in an otherwise normal field of vision.) Intraocular pressure was $12 \mathrm{mmhg}$ bilaterally. Two months later he presented again with further reduction after playing the same piece of music (visual acuity 6/18) (This is Snellen acuity which is measured in metres. $6 / 6$ is roughly equivalent $20 / 20$ in feet). He suffered a nonischemic central retinal vein obstruction in the same eye 10 months later, after playing another strenuous piece, (acuity 6/60). He ended his professional career and had no recurrance in the next 10 years.

Blowing up balloons [7]: A 47-year old man (Case 12) presented with an acute reduction of central acuity in his left eye whilst inflating a long party balloon. He was otherwise well with no past medical or opthalmic history of note. Snellen acuity was 6/24 left eye and 6/6 right eye. Intraocular pressures were $16 \mathrm{mmHg}$ bilaterally. Dilated fundal examination revealed deep and superficial retinal haemorrhages scattered in the posterior pole of the left eye. At 4-week follow-up, visual acuity of the left eye had improved to $6 / 9$ and dilated fundal examination revealed marked resolution of the hemorrhages. At 8 -week follow up, visual acuity had returned to 6/6 in the left eye and the retinal haemorrhages had completely resolved with no evidence of any retinal sequelae.

Valsalva while lifting heavy object [8]: A 43 yr male (Case 13) wore a new back support to help while lifting heavy objects. The left eye demonstrated a vitreous hemorrhage that seemed to originate from the optic disc surface. A number of blood clots were seen in the vitreous below the disc. Intraocular pressures were $16 \mathrm{mmHg}$, then, 1 week later, 13 in each eye. Resolved by one month. 
Aerobic Exercise [9]: A 19-yr-old white female (Case 14) complained of a "dark spot" in her left eye's field of vision. It had appeared 1.5 days earlier- just after completing "bend and reach" exercises during her daily aerobics workout ("Bend and reach" involves changing from a legs apart standing position to a bending position, sufficiently low to reach through between the legs with both arms. This results in repeated compression of the abdomen). Initial symptoms were a slight dizziness followed by the perception of a central darkish haze similar to the after image sensation one experiences when dazzled by a flash bulb.

\section{Visual acuities were 20/15 right and 20/30 left; BP 112/82.}

Posterior segment evaluation of the left eye disclosed a wellcircumscribed retinal hemorrhage near the fovea. At 1 week follow up, there was a slight decrease in left eye's vision to 20/40. After 2 weeks it had improved to 20/25. At 6 weeks acuities were 20/15 right; 20/15, left.

Valsalva retinopathy induced by labour [10]: A 29 yr old (Case 15) lost vision in left eye during labour. Delivery by caesarean section under general anaesthesia was performed to avoid further Valsalva episodes. Fundoscopy was unremarkable in the right eye but in the left eye there was a large, dense, round preretinal hemorrhage that completely obscured all macular details. The premacular hemorrhage was treated with Nd:YAG Laser pulses $(5 \mathrm{~mJ})$ placed at the inferior margin of the hematoma's anterior surface. Within two days, visual acuity had improved to $20 / 60$ and the premacular hemorrhage rapidly cleared. Two weeks later visual acuity was 20/20.

The most striking thing illustrated by the above cases is the variability of vulnerability to this disorder. Olympic Weight Lifters lift tremendous weights without going blind yet blowing up balloons, playing the trombone, aerobic exercise involving compressing the abdomen, are documented to occur. A similar variability in vulnerability to Valsalva Retinopathy must be expected in infants.

Hsu [11] points out that whereas the arterial supply to the brain has been widely studied, venous return has been relatively neglected. "Hinderance of principle and collateral cerebral venous outflow will cause elevation of venous pressure, insufficiency of cerebral blood flow, increase of intracranial pressure, and eventually lead to parenchymal abnormalities" ...."The Internal Jugular Vein is a main extracranial route for venous drainage. The Jugular venous valves, which are located near the junction with the innominant vein, prevent transmission of thoracic pressure and the reflux of venous blood into the cerebral circulation. Jugular valve insufficiency could result in retrograde jugular venous flow and back transmission of central venous pressure".

A second feature of these cases is spontaneous recovery. In many cases, although haemorrhages have been observed by ophthalmography, normal, or near normal acuity returned after a few months. It would seem that some infants may suffer Valsalva Retinopathy but may similarly be asymptomatic after a short while.

\section{Intracranial venous hypertension generation}

The American Academy of Pediatrics' document defining Shaken Baby Syndrome in 2001 [12] states that:- "A victim of sublethal shaking may have a history of poor feeding, vomiting, lethargy, and/or irritability occurring for days or weeks". Since shaking is not involved in Valsalva Retinopathy, perhaps this should read "The injured child may have a history of poor feeding, vomiting, lethargy and/or irritability occurring for days or weeks". These symptoms are typical of digestive tract obstruction by pyloric stenosis.
A basic mechanism generating retinal and cerebral venous hypertension is dealt with in reference [13], an open access document that can be down loaded free of cost from the address indicated. Briefly, in expulsive actions (coughing, Dry Heaves, Vomiting etc.) the diaphragm and abdominal muscles tighten, raising intra-abdominal pressure.

There are no valves in the Inferior Vena Cava (IVC) or Superior Vena Cava (SVC). They both feed into the heart right atrium and there is no valve between them. Limb veins have valves, but there are no valves within the brain. The brain has some protection from excessive SVC pressures by valves in the inner jugular veins (JV in Figure 1) near where they branch off the Brachiocephalic Veins. However, in humans the Inner Jugular vein valves have become optimised for low forward flow resistance, at the expense of merely adequate reverse blocking ability. If sudden excessive venous hypertension occurs, such as in vomiting, the Jugular Vein Valves may fail at a lower pressure than the limb valves (Hamon and Edwards [14] found that out of 100 adult autopsy cases, valves were actually absent in the internal jugular veins of 9 cases that had lived apparently normal lives). If inner jugular vein valves are absent or inadequate retrograde flow of high pressure venous blood into all brain veins will occur.

\section{Cerebral venous configuration}

Whereas the layout of arteries is determined by predetermined genetic factors that of veins "happens" in utero under dissolved gas gradients. As a result the paths of veins vary much more from person to person than arteries do [15]. Figure 2 shows a typical configuration.

Suppose a pressure surge arrives in the Superior Vena Cava (Figure 2), sufficient for reflux venous blood to break through the jugular vein valves. Pressurised venous blood will then flow up the jugular veins, the transverse Sinuses and round to the Torcular, where left and right transverse sinuses usually meet, at the back of the head. The Superior Sagital Sinus usually drains into the torcular and some of this reflux blood will be driven back up into it. The left and right Cavernous Sinuses normally collect blood from the front of the head including the retinal veins. Blood from each cavernous sinus normally drains

\section{RELEVANT VENOUS INTERCONNECTIONS}

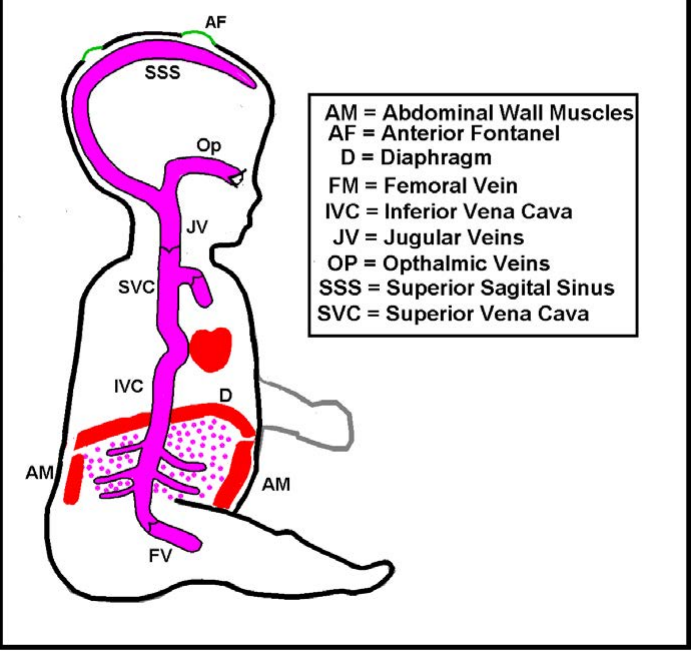

Figure 1. Venous Hypertension. Structures involved in the generation of venous high pressure in vomiting and coughing. 


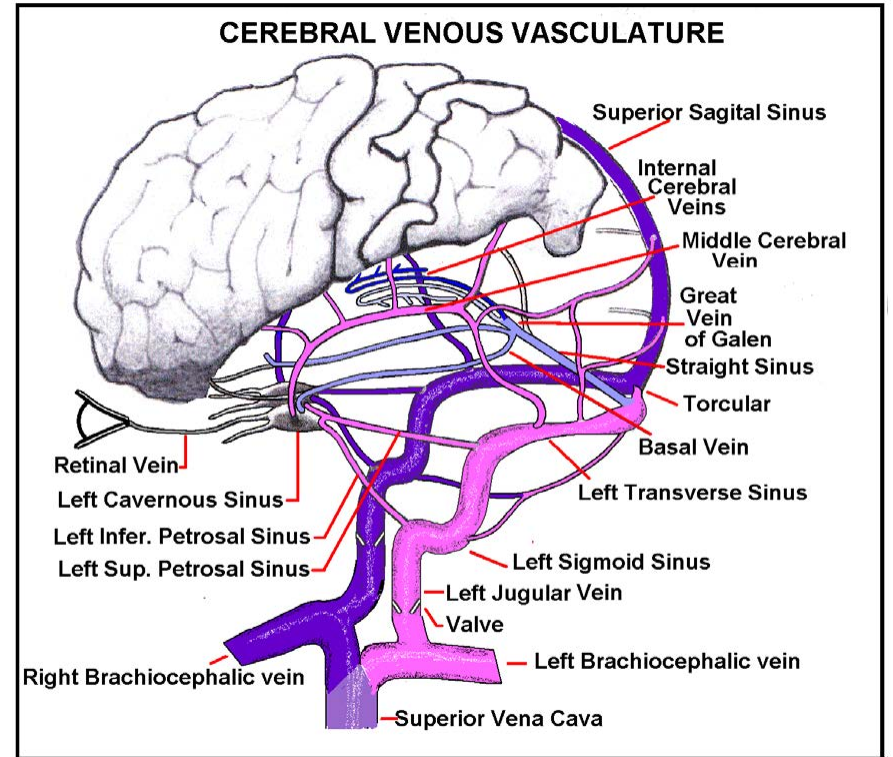

Figure 2. Intra-Cranial Venous Drainage Routes. If Jugular vein valves are weak a pressure surge in the SVC will reverse flow in intracranial veins. Reverse flow through the Jugular veins, Inferior and Superior Petrosal Sinuses, into the Cavernous Sinus, will raise pressure in it to surge pressure.Retinal vein pressure will follow.

into its transverse sinus through its superior petrosal sinus, and into its internal jugular vein via its inferior petrosal sinus [16]. Reverse jugular vein flow will rapidly fill each Cavernous Sinus to the surge pressure. The Central vein of the Retina usually enters its Cavernous Sinus directly so the intraocular Retinal Veins will also rapidly be subject to the full surge pressure.

There is no reason for both Jugular Vein Valves to have the same break-through pressure so unilateral damage may be common. Of course, if the surge is high enough it will overcome both valves and damage will be bi-lateral.

\section{The orbital (extra Ocular) vasculature}

There are two circulations in the eyeball, the Retinal and the Uveal [17]. The retinal layer is supplied by the retinal artery and vein which run along the centre of the Optic nerve, figure 3. These are the vessels that can be seen on the inner surface of the retina in ophthalmology. The rest of the eyeball is supplied by the Uveal circulation (The term "Uveal", Gr. Grape-like was given because its principle component, the Choroid, resembles the skin of a red grape surrounding the flesh of the grape, the retina). The uveal arteries are branches of the opthalmic artery and enter the eyeball without passing with the optic nerve.

The vorticose veins, which drain the rest of the eyeball also drain into Cavernous Sinus via the Opthalmic veins. So any SVC pressure surges sufficient to overcome the Jugular Vein Valves will simultaneously affect both the retinal and uveal veins.

If this venous hypertension surge is sufficient to rupture capillary or venule walls blood will be driven into the virtual space between the retina and the limiting membrane producing a dome shaped hematoma (Figure 7).

\section{Retinal structure and blood supply}

Both retinal circulation and uveal circulations are essential for sight. The best known is the retinal which lines the inner surface of the eyeball wall, fed and drained by the retinal artery and vein which enter and leave the eyeball within the Optic Nerve (Figure 3).

The other is provided by the uveal system via the choroid capillaries which drain into the vorticose veins. The uveal circulation is somewhat complex but highly relevant to retinal detachment. The wall of the eye can be considered as divided into several layers (described by Gray as tunics [18], Figure 4).

The outer layer, the sclera, the white of the eye, is a tough fibrous structure to which external muscles are attached to move the eye.

Next inside the sclera is a vascular layer, known as the choroid. The choroid is firmly attached to the pigmented (outer) layer of the retina inside it. Large arteries and veins run in the outer aspect of the choroid, feeding a dense capillary plexus in its inner aspect known as the choriocapillaris. The choriocapillaris consists of a single layer of unusually wide fenestrated capillaries; these are the only capillaries in the choroid. Their functional importance is that they nourish the outer third of the retina, which is where the photoreceptors are located. In regions where substantial areas of this capillary circulation have been lost as a result of disease, the overlying areas become atrophied [19].

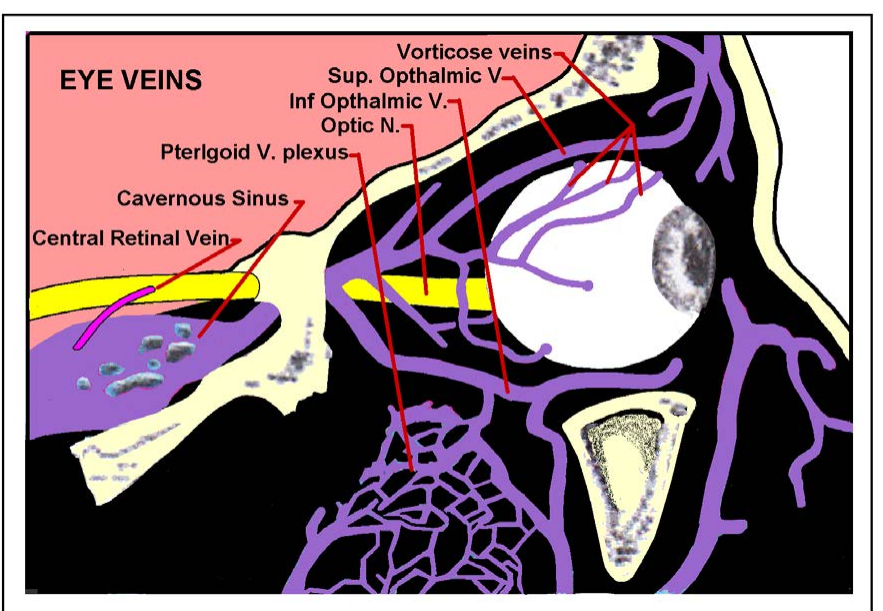

Figure 3. Ocular veins Blood leaving the retina travels through the Central Retina vein within the Optic Nerve.After the nerve passes into the cranium through apertures in the lamina cribrosa, the central retinal vein emerges from the nerve and drains into the Cavernous Sinus.The rest (Uveal veins)mainly drain through the Vorticose Veins into the Opthalmic Veins, and thence through the Cavernous Sinusand Pterigoid venous plexus into a transverse or sigmoid sinus.

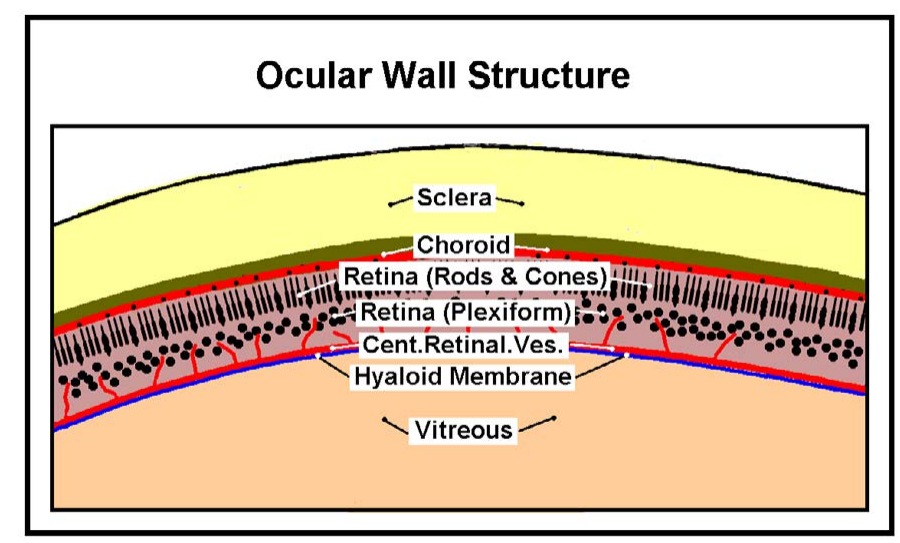

Figure 4. Ocular Wall Structure. The relative positions of principle layers of the Ocular Wall Structure. (Diagramatic; the thicknesses are not to scale). 
Next inside the choroid is the Retina which detects the image focused on it by the Cornea and Lens. It also does some preprocessing to combine signals from over 100 million photocells into the 1.2 million or so myelinated fibres in the optic nerve to the brain. There are two sorts of photoreceptors (photocells), those whose sensing ends (segments) are like rods and those shaped like cones (Figure 5).

The rods are more sensitive than cones, but cones can sense colour. Light has to pass through their bodies and those of some interconnecting neurons, ganglia, capillaries etc. to get to the sensing outer segments. Little light is lost though, because all these cell bodies are largely water and are only micrometers thick. Where it is necessary to absorb scattered light, which would otherwise "blind" neighbouring photocells, local cells produce the pigment "melanin". To maximise resolution the sensing segments are packed much closer than their bodies would allow, so the bodies are packed in random order below the sensing region (the external nuclear layer). In a circle at 10 degrees around the macula the sensing element concentration is about 160,000 per square $\mathrm{mm}$ [20]. This corresponds to one rod sensor every $2.5 \mathrm{um}$, too close for an erythrocyte (Figure 5), to pass between them. Instead, the choroid capillaries lie against the back of the retina, bathing the sensors in rapidly changing serum. These specialised choroid capillaries are fenestrated, that is they have numerous holes in their walls which allow fluids to pass in and out, but retain erythrocytes, platelets etc [21]. Normally the presence of haemoglobin increases the $\mathrm{O}_{2}$ carrying capacity of blood 70-fold... [22]. The oxygen and $\mathrm{CO}_{2}$ carrying power of serum is only that of its water, so this flow has to be copious.

\section{Sheffield retinal injury distribution}

When, in the Sheffield study, the percentage incidence of retinal lesions (subhyaloid haemorrhage and local retinal detachment) in infants diagnosed as dying of non-accidental head injury, were plotted against position in the eyeball, figure 6 , Green et al. found that the incidence of both these lesions is lowest at the equator of the eye, but is almost doubled at the periphery of the retina/ora serrata junction and is increased (but less so) posteriorly around the optic disc and in relation to the macula [1].

Valsalva Retinopathy explains the distribution of haemorrhages in terms of the vasculature. The central arteries and veins of the retina

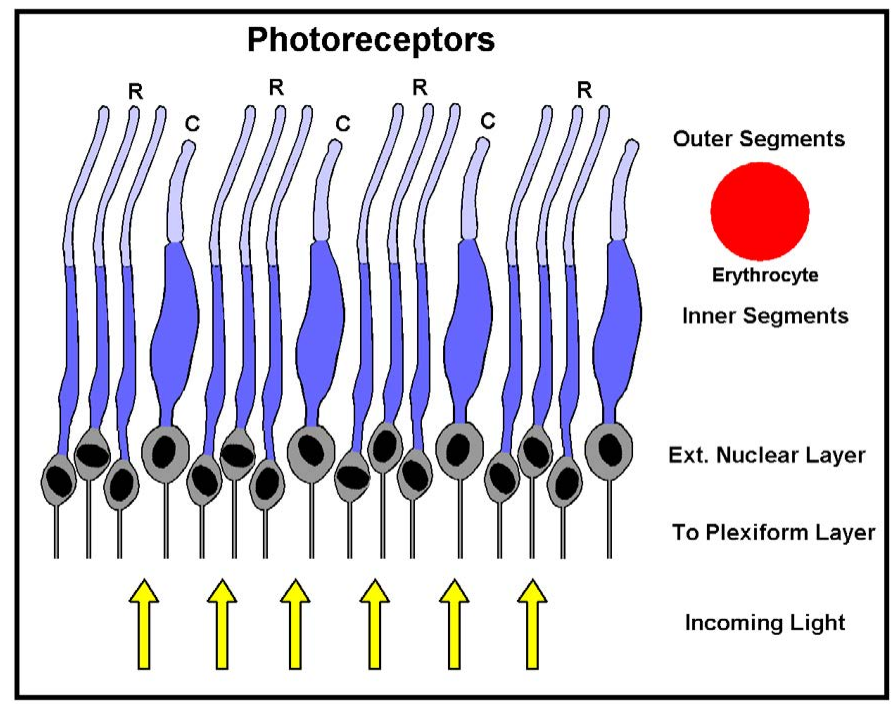

Figure 5. Photocell configuration (detail of figure 4) $R=$ Rods: $C=$ Cones; Erythrocyte to same scale.

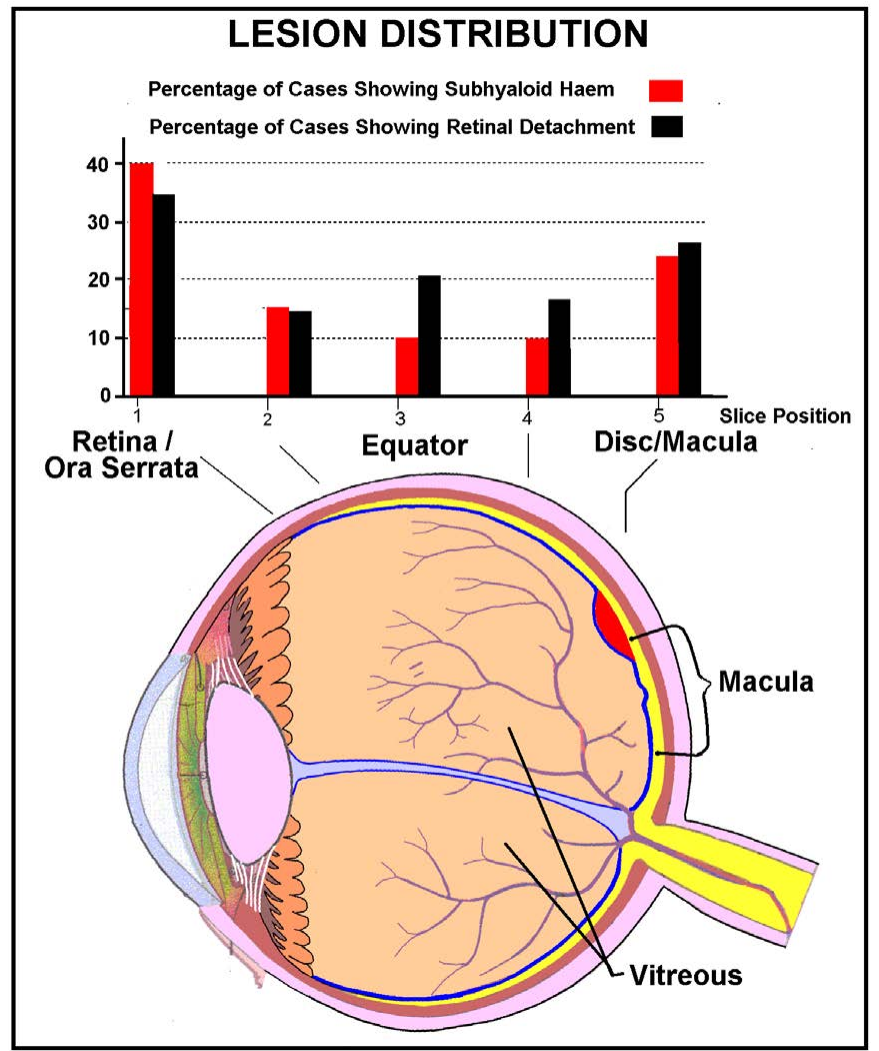

Figure 6. Incidence (\% findings) of subhyaloidhemorrhage and local retinal detachment in each of 5 positions in each eye.

Drawn from figure 1 in reference 1.

Blue=Internal limiting membrane (ILM) $[23,24]$

Red = Illustrative position of a Subhyaloid (preretinal) hemorrhage partly overlapping the Macula

comprise an end artery system, i.e. they do not anastomose. In general the relative sizes of mother and daughter vascular branches develop to minimise energy loss in the circulation [25]. This is reflected in the thickness of the retina. The retina is very thin, varying from 0.56 $\mathrm{mm}$ near the optic disc to $0.1 \mathrm{~mm}$ anterior to the equator of the eyeball, continuing at this thickness to the ora serrate [26].

The lumen pressure a vessel can withstand is related to the maximum tension its walls can withstand by the relationship:-

$$
\mathrm{P}=\mathrm{T} / \mathrm{r}
$$

Where $\mathrm{P}$ is the maximum safe lumen pressure, $\mathrm{T}$ is the tension in the vessel wall, and $r$ is the radius of the vessel, in suitable units (The Law of Laplace $[27,28])$.

That is, for a given vessel structure, the smaller the radius of the vessel the greater the pressure it can withstand. So the venules at the periphery of the retinal circulation can withstand larger pressures than those of similar wall structure nearer the disc. Moreover, capillaries do not actually extend to the Ora Serrata.

The retinal capillaries... are especially numerous in the macula, but absent from the central part of the fovea; they become less numerous in the peripheral retina and are absent from a zone about $1.5 \mathrm{~mm}$ wide adjoining the ora serrate [20]. They are unlikely therefore to be able to produce the highest incidence of haemorrhages at the retina/ora serrata seen in figure 6 in response to pressure surge, or to shaking. 


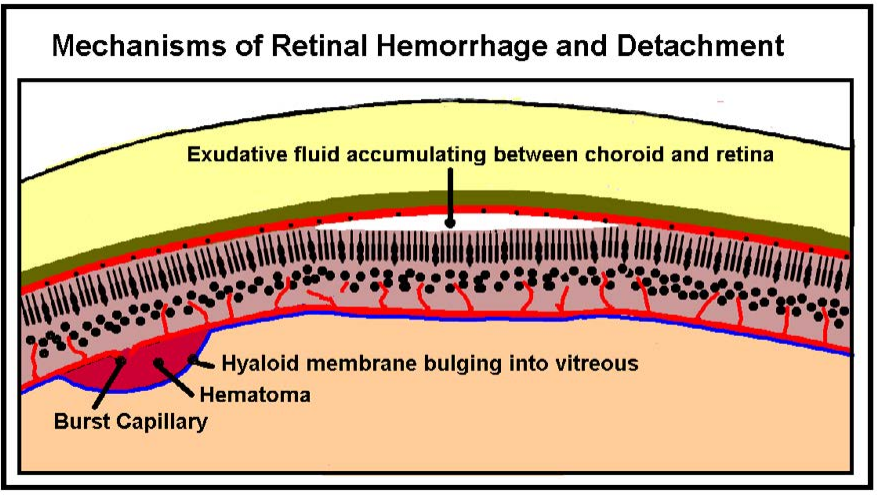

Figure 7. Retinal Hemorrhage and Detachment. If a retinal vein ruptures during a venous pressure surge it may leak retinal blood out under the hyaloid membrane pushing it out into the vitreous. Raised pressure in the choroid veins may force fluid from the choroidal capillaries between the choroid and retinal layers forcing them apart (detachment).

\section{Detachments}

Three forms of detachments are recognised [29].

Rhegmatogenous occurs due to a break in the retina (Retinal Tear) that allows fluid to pass from the vitreous space into the subretinal space between the sensory retina and the retinal pigment epithelium.

\section{Exudative, serous, or secondary}

It occurs due to inflammation, injury or vascular abnormalities that results in fluid accumulating underneath the retina, without the presence of a hole tear or break.

Tractional occurs when fibrous or fibrovascular tissue, caused by an injury, inflammation or neovascularisation, pulls the sensory retina from the retinal pigment epithelium.

Green et al. believed that inertia/momentum of the vitreous body within the eye during shaking caused traction detachments. They reasoned:- "Our findings on plotting the exact site of the retinal lesions, which show that subhyaloid haemorrhage and retinal detachment are most frequent at the periphery of the retina, and posteriorly in relation to the optic nerve, support this hypothesis as these are the precise sites at which the vitreous is most strongly attached to the retina."

Momentum can play no part in Valsalva Retinopathy because movement does not occur. Valsalva Retinopathy suggests an exudative form. The choroid vessels that feed the photodetectors drain ultimately into the cavernous sinus so they will experience any venous hypertensive surge. Surge pressure, driving fluid out of the choroid capillaries into the space between the choroid and retina will be high during a surge. However, the pressure returning the excess fluid is relatively weak. Fluid will accumulate behind the retina, eventually pushing it away from the choroid (Figure 7). This process will be accelerated by the fact that the choroid capillaries "feeding" the photodetectors are fenestrated to produce rapid turnover of fluid.

Green et al. [1] found that the highest incidence of haemorrhages and detachments was at the "periphery of the retina". But this is the region of smallest retinal vessels. Also, the periphery of the retinal circulation is $1.5 \mathrm{~mm}$ behind the Ora Serratta [18]. This suggests that these haemorrhages do not originate from the retinal circulation but from that supplying the muscles of the iris and focusing mechanisms, at the cilio-choroidal junction. There have also been reports of bilateral cilio-choroidal detachments occurring after the Valsalva maneuover [8].
The ciliary muscles are continuously active and so need a plentiful blood supply but flow is greatly in excess of metabolic demand. Choroidal venous blood has only lost $3 \%$ of its oxygen [18] compared to $97 \%-75 \%$ $=22 \%$ body average [22]. It has also been suggested that a copious, fast flow also allows the eye to operate in ambient temperatures below freezing. This ciliary circulation drains largely through the vorticose veins, the ophthalmic veins, and into the Pterigoid venous plexus and Cavernous Sinus (Figure 3). If a venous pressure surge breaks through the valves in an inner jugular vein it will arrive in the ciliary circulation.

The ciliary epithelium is bilaminar, consisting of two layers of simple epithelium superimposed one upon another and representing the two layers of the optic cup. The two layers are firmly united but pathological accumulations of fluid may separate them, just as the retina detaches from its own pigment epithelium [18].

It seems very likely that the cause of detachment at the ora-serrata is seepage of fluid and /or blood from the ciliary circulation.

\section{CNS and ocular injuries are not independent variables}

It is currently assumed that CNS and Ocular injuries are independent variables. Green et al reasoned along the following lines [1].

Although all of the children with perineural optic nerve (sheath) haemorrhages had co-existing subdural haemorrhages, the perineural haemorrhages involved only the distal segment of the nerve, and did not involve the proximal part of the optic nerve within the apex of the orbit, confirming that there was no direct connection between these two separate sites of haemorrhage. ...All patients with perineural optic nerve haemorrhage had coincident intracranial subdural haemorrhages... Examination of only the anterior half of the intraorbital optic nerve might lead to the conclusion that these intracranial and perineural subdural haemorrhages were related. However, we found... that there was no perineural optic nerve haemorrhage at the apex of the orbit, and conclude that perineural optic nerve haemorrhage is a separate entity in these children, and not the result of direct tracking of intracranial subdural haemorrhage into the optic nerve sheath.

However, Valsalva pressure considerations would suggest that during a venous pressure surge the proximal retinal vein cannot dilate where it is supported within the optic nerve, but where it enters the intraocular space the distal part of it is surrounded by intraocular pressure (a few $\mathrm{mmHg}$ ), as are all the veins it drains. Its walls receive little support. This is where distension and rupture will occur during surges. However this has no bearing on the relationship between CNS and ocular injuries, in Valsalva the link is that both are responding to the same intracranial venous pressure surges.

\section{Triad validity}

The term "Subdural Hematoma" is incorrect, cleavage occurs in a layer in the base of the dura [30]. This is a layer whose structure (absence of collagen) suggests its function is to accommodate changes of brain proportion relative to the skull eg: cortex expansion in late pregnancy [31]. The lack of collagen renders it relatively vulnerable to distension by edemous fluid expressed from the bridging veins when they are subjected to venous pressure surges. If this distension becomes excessive bridging veins will be torn, producing haemorrhages. Like Valsalva retinopathy "subdural" haemorrhages are produced by venous hypertension. Moreover, cerebral venous hypertension will be expected to cause cerebral malfunction.

So, Subdural Hemorrhage, Retinopathy and Encephalopathy are 
not independent variables, they are all linked as manifestations of one variable, venous hypertension.

\section{Conclusion}

In 2005 Lord Justice Gage [32] (in the Supreme Court of Judicure, Court of Appeal, England ) declared the state of knowledge of Shaken Baby Syndrome as follows." At the heart of these appeals ....was a challenge to the accepted hypothesis concerning "shaken baby syndrome" or, as we believe it should more properly called, non-accidental head injury. The accepted hypothesis depends on findings of a triad of intracranial injuries consisting of encephalopathy (defined as disease of the brain affecting the brain's function); subdural haemorrhages (SDH); and retinal haemorrhages $(R H)$. For many years the coincidence of these injuries in infants (babies aged between 1 month and 2 years) has been considered the hallmark of NAHI."

The Court judgement that "although the evidence of the findings of retinal haemorrhages is powerful supporting evidence of shaking, on its own, it is not diagnostic of shaking." is fully justified. There are a multitude of situations in which retinal haemorrhages will occur as a result of raised thoracoabdominal pressure.

Vulnerabilty to this form of retinopathy varies with the integrity of the infant's jugular vein valves protecting the brain from excessive SVC pressure surges.

Encephalopathy, SDH and RH are not independent variables, and should not be considered so in the Triad. They are manifestations of a common factor, transient venous hypertension. Their coincidence has no significance.

Infant Valsalva Retinopathy provides a natural explanation of eye injuries currently attributed to criminal imposed trauma by the Shaken Baby Syndrome hypothetical mechanism.

\section{References}

1. Green MA, Lieberman G, Milroy CM, Parsons MA (1996) Ocular and cerebral trauma in non-accidental injury in infancy: underlying mechanisms and implications for paediatric practice. Br J Opthalmol 80: 282-287.

2. Duane TD (1972) Valsalva hemorrhagic retinopathy. Trans Am Ophthalmol Soc 70: 298-313.

3. McKendrick GDW (1978)Bordetellainfections; Whooping cough. In: Scott RB(Ed.), Price's Textbook of the Practice of Medicine. (12thedn), Oxford: Oxford University Press; 49-51.

4. Kocak N, Kaynak S, Kaynak T, Oner HF, Cingil G (2003) Unilateral Purtscher-like retinopathy after weight-lifting. Eur J Ophthalmol 13: 395-397.

5. Bacon CJ, Sayer GC, Howe JW (1978) Extensive retinal haemorrhages in infancy--an innocent cause. Br Med $J$ 1: 281.

6. Sbeity ZH, Mansour AM (2004) Recurrent retinal vein occlusion after playing a wind instrument. Graefes Arch Clin Exp Ophthalmol 242: 428-431.

7. Georgiou T, Pearce IA, Taylor RH (1999) Valsalva retinopathy associated with blowing balloons. Eye (Lond) 13: 686-687.

8. Jones WL (1995) Valsalva maneuver induced vitreous hemorrhage. J Am Optom Assoc 66: 301-304.

9. Roberts DK, MacKay KA (1987) Microhemorrhagicmaculopathy associated with aerobic exercise. J Am OptomAssoc58: 415-418.

10. Ladjimi A, Zaouali S, Messaoud R, Ben Yahia S, Attia S, et al. (2002) Valsalva retinopathy induced by labour. Eur J Ophthalmol 12: 336-338.

11. Hsu HY (2011) Jugular venous reflux and neurological disorders. Acta Neurol Taiwan 20: $1-3$.

12. American Academy of Pediatrics (2001) Shaken Baby Syndrome:Rotational Cranial Injuries-Technical Report. Pediatrics 108: 206-210.
13. Talbert DG (2012) Pyloric Stenosis as a cause of a Venous Hypertensive Syndrome Mimicing True Shaken Baby Syndrome. J Trauma Treat 1:102 http://dx.doi. org/10.4172/2167-1222.1000102.

14. Harmon JV Jr, Edwards WD (1987) Venous valves in subclavian and internal jugular veins. Frequency, position, and structure in 100 autopsy cases. Am J Cardiovasc Pathol 1: $51-54$.

15. Talbert DG (2016) Cerebral Venous Malformation as a Cause of of Neonatal IntraVentricular Haemorrhage and Unexplained Infant Subdural Haemorrhage. Anat Physiol http://dx.doi.org/10.4172/2161-0940.1000202.

16. Gray H, Warwick R, Williams RL (1973) Neurology-(Vasculature). Gray’s Anatomy. (35edn), Edinburgh: Longman; 980.

17. Wikipedia. Choroid. Wikipedia 2016.

18. Gray H, Warwick R, Williams RL (1973) Neurology-The peripheral visual apparatus. Gray's Anatomy. (35edn), Edinburgh: Longman; 1096-1122.

19. Cormack DH (1953)The Eye and Ear. In: Cormack DH (Ed.), Ham's Histology. (9thedn), Lippincott; 678-707.

20. Gray H (1973) Development of Special Sense Organs. Gray. (35edn), 144-149.

21. Cormack DH (1987)Loose connective tissue and Adipose Tissue. Ham's Histology. Philadelphia: Lippincott; 155-187.

22. Ganong WF (1987) Gas transport between the lungs \& the tissues. Review of medical physiology. (13edn), Connecticut: Prentice-Hall International Inc; 551-557.

23. Mennel S (2007) Subhyaloidal and macular haemorrhage: localisation and treatment strategies. Br J Ophthalmol 91: 850-852.

24. Murtaza F, Rizvi SF, Bokhari SA, Kamil Z (2014) Management of Macular Pre-Retinal Subhyaloid hemorrhage by Nd:Yag laser hyaloidotomy. Pak J Med Sci 30: 339-342.

25. Sherman TF (1981) On connecting large vessels to small. The meaning of Murray's law. J Gen Physiol 78: 431-453.

26. Warwick R, Williams PL (2016)The Visual Apparatus. Gray's Anatomy. Edinburgh7: Longman; 1095-1133.

27. Sircar S (2008) General Physiology. In: Sircar S (Ed.), Principles of medical physiology (1stedn.), Stuttgart: Thieme; 3-18.

28. Burton AC (1951) On the physical equilibrium of small blood vessels. Am J Physiol 164: 319-329.

29. Wikipedia (2016) Retinal detachment. Wikipedia.

30. Talbert DG (2014) The Legacy of John Caffey: Shaken Baby or Pyloric Stenosis? $J$ Trauma Treat http://dx.doi.org/10.4172/2167-1222.1000192.

31. Talbert DG (2016) The Subdural Myth: Space or Place? Anatomy \& Physiology: Current Research 6: 5 http://dx.doi.org/10.4172/2161-0940.1000242.

32. Gage LJ, Gross J, McFarlane J (2005) Supreme Court of Judicature, Court of Appeal Neutral citation Number, EWCA Crim 1980 case Nos:200403277,200406902,200405 573,200302848 .

Copyright: (C2017 Talbert DG. This is an open-access article distributed under the terms of the Creative Commons Attribution License, which permits unrestricted use, distribution, and reproduction in any medium, provided the original author and source are credited. 